\title{
VHDL-AMS Model of an Electrochemical Cell to Design VLSI Bio-Chips
}

\author{
Marialaura Beltrandi, Alain Vachoux*, Sandro Carrara,Yusuf Leblebici, Giovanni De Micheli \\ EPFL, Lausanne, Switzerland \\ *alain.vachoux@epfl.ch
}

\begin{abstract}
This paper presents a new VHDL-AMS behavioral model of an electrochemical cell suitable for the design of VLSI bio-chips. Existing models are mainly equivalent circuit macromodels using electrical primitives, which hardly fit the actual behavior of biosensors. The presented VHDL-AMS model expresses a more realistic behavior as it can directly use electrochemical equations. It can also be made very flexible and reusable for different experimentation cases. Last, but not least, it can be easily used for the design of bio-chips.
\end{abstract}

\section{INTRODUCTION}

Redox reactions are detectable by using electrochemical techniques such as cyclic voltammetry or chronoamperometry [1]. Redox reactions involving proteins such as oxidases of cytochromes are of crucial importance to develop new technology addressing the human metabolism monitoring [2] and to develop new technologies for regenerative medicine [3]. The integration of these biological molecules with nanostructures, such as carbon nanotubes, is a crucial aspect to improve the device performances for glucose detection [4], and more in general for oxidase or cytochrome based detection. The further integration of these nano-bio-chemical sensors with the CMOS technology [5] is the final step to realize platforms for advanced diagnostics [2]. However, despite simple solutions are available to realize VLSI versions of a potentiostat [6], the simulation models used to verify the performances of the proposed architectures are, more often, too simplistic [6]. The aim of the present paper is to propose a new behavioral simulation model realized in the VHDL-AMS modeling language, which seamlessly integrates in the VLSI design flow.

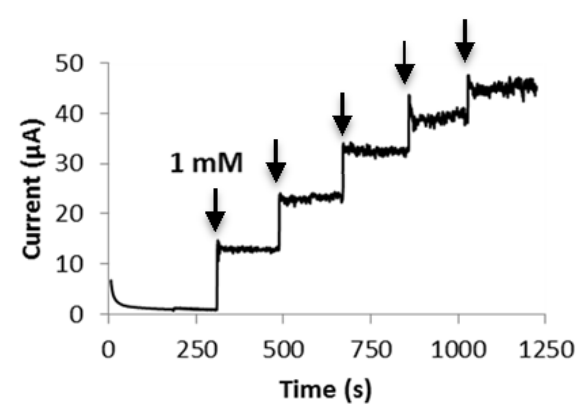

Figure 1. Chronoamperometry of SPE at potential of $+650 \mathrm{mV}$ with 5 subsequent additions of $1 \mathrm{mM} \mathrm{H}_{2} \mathrm{O}_{2}$ in PBS on a working electrode of $13 \mathrm{~mm}^{2}$ of area.

This research has been supported by the i-IronIC project, financed with a grant from the Swiss Nano-Tera.ch initiative and evaluated by the Swiss National Science Foundation. The research has also been funded by the project NanoSys, within the program ERC-2009-ADG-246810.

\section{THE AMPEROMETRIC DETECTION}

The electrochemical cell is the core of every circuit for bio-sensing, to which one can apply a potential, with the aim of measuring a resulting current that is related to the concentration of the target species to be detected. In this context, enzymes are very useful proteins that play an important role in the detection of both endogenous (such as glucose, lactate, glutamate and cholesterol) and exogenous molecules (drug compounds). In particular, the protein class of oxidases, mostly used for sensing endogenous metabolites [2], catalyzes an oxidation-reduction reaction, that has water $\left(\mathrm{H}_{2} \mathrm{O}\right)$ and hydrogen peroxide $\left(\mathrm{H}_{2} \mathrm{O}_{2}\right)$ as final products. Hydrogen peroxide is an electrochemical active species, which oxidizes according to the following reaction, which can be promoted by applying a potential to the electrochemical cell:

$$
2 \mathrm{H}_{2} \mathrm{O}_{2} \rightarrow 2 \mathrm{H}_{2} \mathrm{O}+\mathrm{O}_{2}^{+}+4 e^{-}
$$

So, the oxidation of each $\mathrm{H}_{2} \mathrm{O}_{2}$ molecule results in a transfer of two electrons to the electrode. This electron transfer is highly enhanced by the presence of multi-walled carbon nanotubes (MWCNT) used as electrons mediators at the Working Electrode by enlarging the surface area, reducing the redox potential, and improving the electrode transfer [3]. As shown by cyclic voltammetries in literature [3], the oxidation peak of hydrogen peroxide is located at a potential around $+650 \mathrm{mV}$ (vs. silver chloride Reference Electrode): it means that the four electrons released to the electrode are optimally detected at that potential. For this reason, it is possible to apply a constant potential (the oxidation potential) between the Working and the Reference Electrodes, and then measure the current flowing from the Working to the Counter Electrodes, which will be directly proportional to the concentration of the target molecule. This is one of the most exploited techniques for amperometric detection using a biosensor: it is called chronoamperometry, because the current flowing between the working and the counter electrodes is monitored as a function of time (Fig. 1).

\section{A. The Cottrell equation}

The most significant equation that describes the behavior of the current in an electrochemical cell to which a constant potential has been applied, is the so called Cottrell equation:

$$
i([C], t)=\frac{n \cdot F \cdot A \cdot \sqrt{D} \cdot[C]}{\sqrt{\pi} \cdot \sqrt{t}}
$$


where $n$ is the number of transferred electrons, $F$ is the Faraday's constant, $A$ the electrode area, $D$ the diffusion coefficient of the oxidized species and $[\mathrm{C}]$ the concentration of the target molecule. It's easy to notice that a linear relationship between the current and the concentration exists: the slope of the curve that one can obtain plotting $i$ vs. [C], the so called calibration curve [3], gives the sensitivity, $\beta$, of the sensor, defined as:

$$
\beta=\frac{\Delta i}{\Delta[C]}
$$

As shown by Eq. (2), the current presents also a specific dependence on time: at given constant applied potential, the current decays as the inverse of the square root of time. Assuming to be under small $A / V$ conditions, that means the electrode area, $A$, is small enough, and the solution volume, $V$, is large enough, that the passage of current does not alter the bulk concentrations of electro-active species, we can well approximate to have an almost constant concentration of the target molecule, after an addition of a certain amount of substrate.

\section{B. Estimation of the diffusion coefficient of $\mathrm{H}_{2} \mathrm{O}_{2}$}

Since we focused our attention on the detection of hydrogen peroxide, which is also produced during enzymatic reaction of oxidases, e.g., glucose oxidase (GOD) and lactate oxidase (LOD), in presence of their relative substrates (glucose and lactate), it has been important to provide a good estimation of its diffusion coefficient, in order to efficiently employ Eq. (2) inside our model. The $\mathrm{H}_{2} \mathrm{O}_{2}$ molecule, whose spatial organization is well known, has been considered as a spherical particle with a radius almost equal to the covalent radius of oxygen $(\approx 70 \mathrm{pm})$. For this reason we could exploit the so called Einstein-Stokes relation, which offers a way to calculate the diffusivity of spherical particles through a fluid medium with dynamic viscosity $\mu$ and low Reynolds number. This relation is defined as follows:

$$
D=\frac{K_{B} \cdot T}{6 \cdot \pi \cdot r \cdot \mu}
$$

where $K_{B}$ is the Boltzmann's constant, $T$ the absolute temperature and $r$ the radius of the molecule.

\section{EXPERIMENTAL DATA}

Looking at the equations presented previously, we can notice that the theory is missing the actual dependency of the resulting current on the value of constant potential that is applied to an electrochemical cell during chronoamperometry experiments. In order to solve this issue, a number of experiments were performed, with the aim of obtaining the "missing equation", which will allow us to develop a complete and accurate model of the real behavior of the biosensor.

Considering the same experimental conditions presented in a previous work [3], eight chronoamperometries, each one with five steps of concentration addition, were performed at different potentials, from +200 to $+900 \mathrm{mV}$ by step of 100 $\mathrm{mV}$. In addition, a chronoamperometry at the potential of $+650 \mathrm{mV}$ was carried out.

\section{A. The missing equation}

The literature of electrochemistry [1] provides some equations that allow us to calculate, for example, the interfacial potential difference of an electrode as a function of the concentrations of the oxidized and reduced forms of the species involved (Nernst equation) and the behavior of the current in time at a given concentration of substrate (Eq. 2). The problem is that all these equations do not supply a way to study the behavior of the current if, for instance, the constant applied potential is moved to a different value. In other words, the literature is missing a relationship for chronoamperometry between the current and the applied potential at a given substrate concentration. That is the main reason why the experiments, mentioned above, were performed. Analyzing all the data acquired during the chronoamperometric measurements and then plotting the mean values reached by the current for a fixed value of hydrogen peroxide concentration over the range of different potentials, a sigmoidal behavior like that shown in Fig. 2 was obtained. This kind of current-voltage curves is usually known under the name of hydrodynamic voltammograms (HDVS) [4]. By means of a statistical fitting of the obtained experimental data, it is possible to define the following equation:

$$
I([C], V)=\beta \cdot[C] \cdot \frac{1}{1+e^{-\alpha \cdot\left(V-V_{0}\right)}}
$$

where $\beta$ is the sensitivity of the biosensor, expressed in $A \cdot m M^{-1}, \alpha$ is a scale factor, in $V^{-1}$, determining the shape of the sigmoidal curve and $V_{0}$ is the potential on which the curve is centered.

Thanks to a thorough data analysis, a further dependence of $\beta$ on the potential was discovered: a large variation of the sensitivity can be easily noticed looking at Fig. 3, which shows how the slope of the calibration curves changed when the potential was moved to different values. This $\beta$ - $V$ dependence could be well approximated by a linear relationship and so $\beta$ was defined as:

$$
\beta=\gamma \cdot V
$$

where $\gamma$ is the proportionality constant expressed in $A \cdot m M^{-1} \cdot V^{-1}$. The fitting operation gave a value of $\gamma$ around $24 \mu \mathrm{A} \cdot \mathrm{m} M^{-1} \cdot V^{-1}$. On the other hand $\alpha$ seemed to be almost constant in the HDVs for all the concentrations considered and its value was always around $12 \mathrm{~V}^{-1}$. Every HDV was also centered almost on the same potential, around $+550 \mathrm{mV}$, so $V_{0}$ can reasonably assume this value.

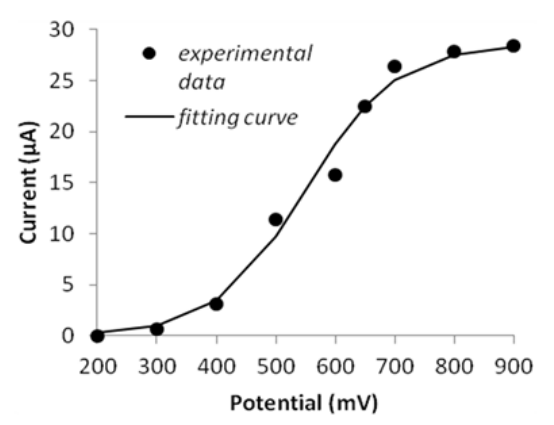

Figure 2. Hydrodynamic voltammogram for $2 \mathrm{mM} \mathrm{H} 2 \mathrm{O} 2$ in PBS. 


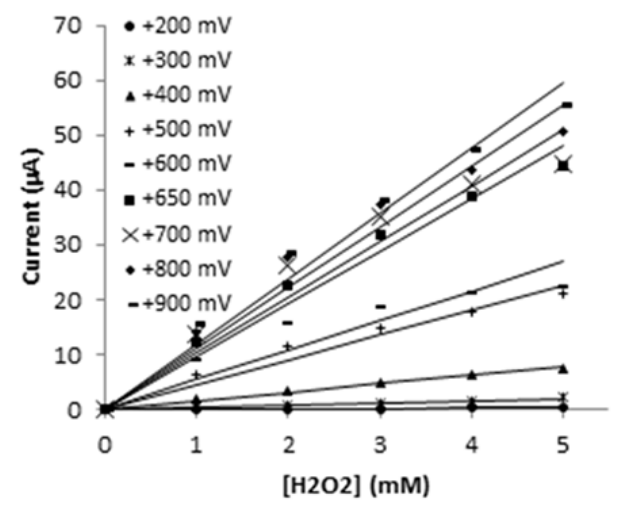

Figure 3. Calibration curves at different potentials from +200 to $+900 \mathrm{mV}$ for $\mathrm{H} 2 \mathrm{O} 2$ in PBS.

\section{THE VHDL-AMS MODEL}

For realizing a three-electrode measurement system, an electronic instrument, called potentiostat, is required to control the potential difference between the working and the reference electrode [5]. To properly design a potentiostat circuit that reaches a high level of stability, a deep understanding of the electrical behavior of the electrochemical cell is necessary. To that end, simple equivalent circuits have been first developed. The basic model and usually the starting point for modeling most electrode-solution interfaces is the Randles' equivalent circuit [6], which is basically made of two resistances, one capacitance and one general impedance. Another equivalent circuit also includes an active element in the form of an ideal current source that models the current generated due to the chemical reaction at the working electrode [7]. The problem with this kind of models is that it is very hard to really fit the equations of the biosensor's behavior onto an equivalent circuit macro-model using primitives like resistors, capacitors and current sources, resulting in strong limitations in the use of these models.

The selected VHDL-AMS modeling language is an IEEE 1076.1 standard [8] that primarily supports the modeling and the simulation of analog and mixed-signal (AMS) integrated circuits, but that can be also applied to multi-physics systems [9]. The language provides ways to express ordinary differential equations as well as procedural statements and eventdriven concurrent processes. As such, it is possible to exploit these capabilities to efficiently map the presented equations onto a behavioral model of the electrochemical cell that supports chronoamperometry experiments.

Fig. 4 shows a schematic representation of the model's interface and typical input/output waveforms. Usually, a constant potential $V(t)$ and a concentration of the target molecule $C(t)$ that rises by steps every time an addition of substrate to the solution occurs are applied. The output current $I(t)$ has then the typical step-shaped resulting aspect.

The model's entity declares the inputs and the output as continuous-time quantities. It also declares a number of constant primary parameters, called generic parameters in VHDL-AMS (Table I). The model's architecture defines the intended time-domain behavior. The behavior is parameter-

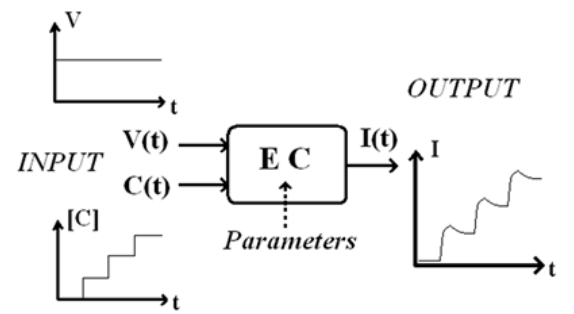

Figure 4. Schematic representation of the port interface of the electrochemical cell behavioral model and its typical use.

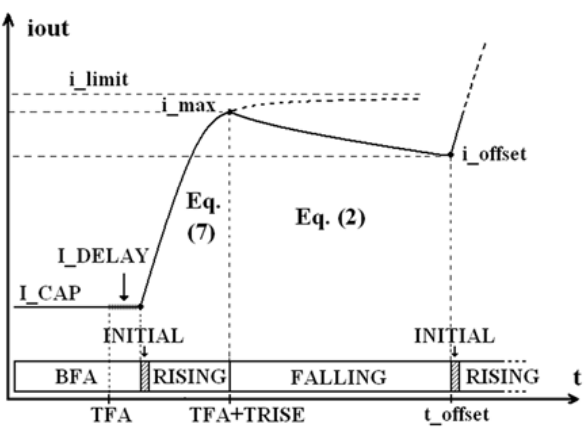

Figure 5. Regions of operation of a single cycle and corresponding behavior of the output current.

ized by the primary parameters and by constant secondary parameters that derive from the primary ones. In our case, only one secondary parameter is needed, namely the diffusion coefficient (DIFF_COEFF) that can be calculated using Eq. (4), which employs the temperature (TEMP), the dynamic viscosity of the medium (MU) and the radius of the target molecule (MOL_RAD).

TABLE I. LIST OF PRIMARY PARAMETERS WITH THEIR RESPECTIVE DEFAULT VALUES AND UNITS

\begin{tabular}{|r|l||r|l|}
\hline Name & Default Value & Name & Default Value \\
\hline TEMP & $300[\mathrm{~K}]$ & TRISE & $10[\mathrm{sec}]$ \\
\hline FAR & $96485.3415[\mathrm{C} / \mathrm{mol}]$ & I_LIM_ERR & $0.01[-]$ \\
\hline A_EL & $12.65 \cdot 10^{-6}\left[\mathrm{~m}^{2}\right]$ & I_CAP & $10^{-6}[\mathrm{~A}]$ \\
\hline MU & $10^{-6}[\mathrm{~kg} /(\mathrm{m} \cdot \mathrm{sec})]$ & VIN_TOL & $0.01[-]$ \\
\hline N_EL & $2[-]$ & CONC_TOL & $0.01[-]$ \\
\hline MOL_RAD & $0.7 \cdot 10^{-10}[\mathrm{~m}]$ & V0 & $550 \cdot 10^{-3}[\mathrm{~V}]$ \\
\hline TFA & $300[\mathrm{sec}]$ & ALFA & $12.28\left[\mathrm{~V}^{-1}\right]$ \\
\hline I_DELAY & $20[\mathrm{sec}]$ & GAMMA & $24 \cdot 10^{-6}[\mathrm{~A} /(\mathrm{mM} \cdot \mathrm{V})]$ \\
\hline
\end{tabular}

Fig. 5 shows that the behavior is modeled as a set of states or regions of operation, each one considering a particular equation for the output current. The BFA ("Before First Addition") state is the initial state that lasts till TFA ("Time of First Addition") and for which the output current is equal to the constant capacitive current I_CAP. Then, the other states are repeated in that same order for every step of current till the end of simulation.

The INITIAL-RISING-FALLING cycle is triggered every time the input concentration has a significant change, thus when an addition of substrate occurs. The INITIAL state is actually an artificial state that is needed for sampling both the voltage and concentration inputs and computing all the data used by the equations governing the behavior of the current in the next two RISING and FALLING states. During the INITIAL state, the value of the beta sensitivity is calculated 
from Eq. (6) by using the GAMMA parameter and the sampled input voltage, and the value of the step of current due to a certain concentration of substrate $\left(i_{-}\right.$limit) is calculated from Eq. (5), which needs beta, the two sampled inputs and the parameters ALFA and V0. Data about $i$ offset and $t$ offset are computed to ensure the continuity of the curve between two subsequent steps, and the tau time constant of the exponential considered at the next state. In the next RISING state, the steep increase of the current is modeled as a rising exponential as follows:

$$
\begin{aligned}
\operatorname{iout}(t)= & i_{-} \text {offset }+ \\
& \left(I_{-} C A P+i_{-} \text {limit }-i_{-} \text {offset }\right) \cdot\left(1-e^{-\frac{t-t \_o f f s e t}{\text { tau }}}\right)
\end{aligned}
$$

The current actually starts to rise after a delay (I_DELAY) that models the time to react to an abrupt change of concentration. The RISING state lasts for a time given by the TRISE parameter, which is the time interval the current takes to reach the maximum value $(i \max )$. In the last FALLING state of a cycle, the decrease of the current in time is governed by the Cottrell equation (Eq. (2)), which needs the number of transferred electrons $(N E L)$, the Faraday's constant $(F A R)$, the electrode area $\left(A_{-} E \bar{L}\right)$ and the value of the input concentration to be computed. The model stays in this state until a new significant change of the input concentration occurs.

\section{Simulation Results}

In order to observe the actual behavior of the model and therefore to verify its correctness, a simulation of a typical chronoamperometry experiment has been performed with the ADVance MS simulator from Mentor Graphics. The experiment consists of an amperometric detection of hydrogen peroxide with a constant applied potential of $+650 \mathrm{mV}$ using an electrochemical cell, in which 5 subsequent additions of substrate have been made: $\mathrm{H}_{2} \mathrm{O}_{2}$ concentration increases of $1 \mathrm{mM}$ every $3 \mathrm{~min}$, going from 0 to $5 \mathrm{mM}$. Since the first addition is made around 5 min after the beginning of the experiment, the overall duration of the experiment is around $20 \mathrm{~min}$. The model takes about $2 \mathrm{sec}$ to simulate the whole resulting curve on a $2.66 \mathrm{GHz}$ Xeon E5650 processor running Linux RedHat5.

For simplicity, all the primary parameters have their default values shown in Table I. Fig. 6 shows that the obtained waveforms are in good accordance with the real experiment of Fig. 1 (further work will consider adding noise). The concentration dependency is properly considered and the current values are in the right range. Values of primary model parameters (Table I) can be easily changed for other experiment conditions. The model is therefore an excellent support for designing potentiostats.

\section{CONCLUSION}

A new model realized in VHDL-AMS language has been presented in this paper. The theoretical frame that represents the background of any electrochemical bio-detection has been summarized. A set of experimental results in the case of hy-

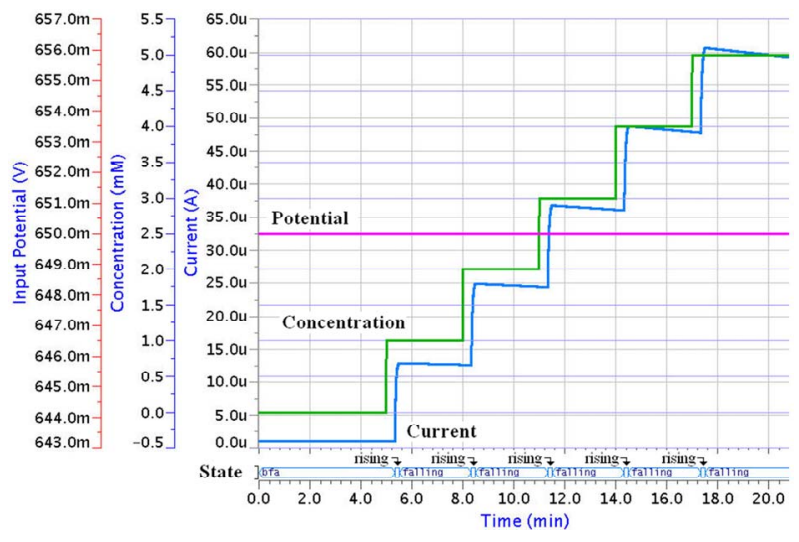

Figure 6. Simulation of the EC behavioral model for chronoamperometry at potential of $+650 \mathrm{mV}$ with 5 steps of concentration addition.

drogen peroxide detection have been proposed to show that a further equation is required to complete this theoretical frame toward the development of a model suitable for designing VLSI bio-chips. Finally, the VHDL-AMS model has been defined and developed. Simulation of this model has demonstrated its suitability as an accurate, yet fast, tool to simulate electrochemical cells in integrated circuit design of bio-chip for detection of metabolites by using the electrochemical technique called chronoamperometry.

\section{ACKNOWLEDGMENTS}

Marialaura thanks Cristina Boero for her support in the acquisition of the experimental data.

\section{REFERENCES}

[1] A.J. Bard and L.R. Faulkner, Electrochemical Methods: Fundamentals and Applications, Wiley, New York, 2001, $2^{\text {nd }}$ Edition.

[2] G. De Micheli, S.S. Ghoreishizadeh, C. Boero, F. Valgimigli, S. Carrara, "An integrated platform for advanced diagnostics", DATE, November 2011, Grenoble, France.

[3] C. Boero, S. Carrara and G. De Micheli, "Sensitivity enhancement by carbon nanotubes: applications to stem cell cultures monitoring", IEEE PRIME, July 2009, Cork, Ireland.

[4] M. Tsai and Y. Tsai, "Adsorption of glucose oxidase at platinummultiwalled carbon nanotube-alumina-coated silica nanocomposite for amperometric glucose biosensor", Sensor and Actuators B: Chem. 141, p. $592-598,2009$.

[5] A. Hierlemann, Integrated Chemical Microsensor Systems in CMOS Technology, Springer-Verlag, Berlin, 2005.

[6] M.M. Ahmadi and G. Jullien, "Circuits for Amperometric Electrochemical Sensors", chapter 13 (p. 265-284) in Krzysztof Iniewski (Ed) "VLSI Circuits for Biomedical Applications", Artech House Inc. Norwood, MA, 2008.

[7] M.M. Ahmadi and G. Jullien, "A very low power CMOS potentiostat for bioimplantable applications", Proceedings of the $9^{\text {th }}$ International Database Engineering \& Application Symposium (IDEAS'05), 2005.

[8] "IEEE Standard VHDL Analog and Mixed-Signal Extensions (IEEE Std. 1076.1-2007)," IEEE Computer Society, 2007, pp. c1-328.

[9] F. Pêcheux, C. Lallement, A. Vachoux, "VHDL-AMS and VerilogAMS as alternative hardware description languages for efficient modeling of multidiscipline systems," IEEE Trans. on Computer-Aided Design of Integrated Circuits and Systems, vol. 24, pp. 204-225, 2005. 